\title{
Granulomatosis polyangiitis associated with meningeal involvement: response to rituximab therapy after failure of cyclophosphamide
}

\author{
M. Benucci' ${ }^{1}$, F. Li Gobbi ${ }^{1}$, P. Panconesi ${ }^{2}$, M. Manfredi ${ }^{3}$, P. Sarzi-Puttini ${ }^{4}$, F. Atzeni ${ }^{4}$ \\ ${ }^{1}$ Rheumatology Unit, San Giovanni di Dio Hospital, Florence; \\ ${ }^{2}$ Radiology Unit, San Giovanni di Dio Hospital, Florence; \\ 3Immunology and Allergology Laboratory, San Giovanni di Dio Hospital, Florence; \\ ${ }^{4}$ Rheumatology Unit, L. Sacco University Hospital, Milan, Italy
}

\section{SUMMARY}

Wegener's granulomatosis or granulomatosis polyangiitis associated (GPA) is a granulomatous inflammation of the upper and lower respiratory tracts associated with necrotising vasculitis of small and medium-sized blood vessels and, frequently, necrotising glomerulonephritis.

We describe the case of a 37 year old female patient presenting with upper respiratory tract involvement, chronic rhinosinusitis with green secretions, and bilateral hypoacusia. Ten months later, she suffered occipital headache and two episodes of lipothymia associated with nausea, photophobia, faintness with visual blurring. Magnetic resonance imaging (MRI) revealed aseptic meningitis. The patient was treated with steroids and cyclophosphamide without any effect on the neurological symptoms which, however, greatly improved after subsequent treatment with rituximab as confirmed by means of cerebral MRI.

Rituximab is an optimal means of treating cyclophosphamide-resistant patients with GPA associated with meningeal involvement.

Key words: Granulomatosis polyangiitis associated, Rituximab.

Reumatismo, 2013; 65 (2): 90-94

\section{INTRODUGTION}

W egener's granulomatosis (WG) or granulomatosis polyangiitis associated (GPA) is a granulomatous inflammation of the upper and lower respiratory tracts associated with necrotising vasculitis of small and medium-sized blood vessels and, frequently, necrotising glomerulonephritis (1). The introduction of glucocorticoid and cyclophosphamide (CYC) treatment about thirty years ago (2) was a major step forward in the management of the disease, which had been almost invariably fatal until then. However, treatment is still unsatisfactory because about $25 \%$ of the patients treated with alkylating agents or methotrexate (MTX) do not achieve complete disease remission (3-5), relapses occur in $15-45 \%(6,7)$, and $20-50 \%$ develop severe treatment-related adverse events $(3,5)$.
Consequently, many new treatments have been evaluated in an attempt to improve efficacy and reduce the toxicity associated with the prolonged use of alkylating agents $(5,8,9)$.

Rituximab (RTX), a chimeric monoclonal anti-CD20 antibody that selectively depletes B lymphocytes for prolonged periods and is used to treat B cell lymphomas, has recently been used to treat rheumatoid arthritis and various refractory autoimmune diseases (10).

\section{CASE REPORT}

In November 2008, a 37 year old female weighing $101 \mathrm{~kg}$ was admitted to our Unit because of mild fever, arthralgia, diffuse abdominal pain, bilateral hypoacusia, right-sided otalgia and cephalea.

Her medical history included a diagnosis 
of hypertension in May 2008, and an acute myocardial infarction treated by means of percutaneous transluminal coronary angioplasty and the placement of a posterior intraventricular artery stent in August 2008. In September 2008, she suffered from blindness in the right eye, and an ocular fundus test revealed occlusion of the central retinal arthery with papillitis. The patient reported chronic rhinosinusitis with green secretions, cultures of which proved to be positive for Staphylococcus aureus. Magnetic resonance imaging (MRI) revealed inflammation of the middle ear bone and sinus opacity. Nasal mucosal biopsy showed a granulomatous inflammation in the wall of arteries or arterioles, in perivascular and extravascular sites as defined by the American College of Rheumatology criteria (11). Laboratory tests showed an erythrocyte sedimentation rate of $78 \mathrm{~mm} / \mathrm{h}$ (normal value (n.v.) $\leq 20 \mathrm{~mm} / \mathrm{R}$ ), C-reactive protein $3.4 \mathrm{mg} / \mathrm{dL}$ (n.v. $\leq 0.5 \mathrm{mg} / \mathrm{dL}$ ), leukocytosis 13,450 white cells $/ \mathrm{mm}^{3}$ and anti-proteinase 3 (anti-PR3) measured by means of immunofluorescence (Euroimmun, Lubeck, Germany) and by combo EIA index (Orgentec Diagnostika GmbH, Mainz, Germany) positive with title 1:160. A diagnosis of limited GPA was made and the patient was treated with prednisone $75 \mathrm{mg} /$ day plus CYC $2 \mathrm{mg} / \mathrm{kg}$, discharged, and then followed-up every three months. Ten months later, in October 2009, the patient suffered from persistent occipital headache and two episodes of lipothymia associated with nausea, photophobia, faintness and visual blurring. Brain MRI revealed an increased gadolinium signal indicating dural thickening in the tentorium of the cerebellum (Fig. 1).

The patient was treated with pulsed methylprednisolone $1 \mathrm{~g} /$ day for two consecutive days and pulsed CYC $1 \mathrm{~g}$ every 15 days, which was stopped after 3 months due to the increase of headache associated with nausea and photophobia and possible adverse events due to the cumulative dose of CYC (6 g).

A screening for hepatitis B and C virus, tuberculosis including Mantoux, Quantiferon tests and chest radiography was negative.

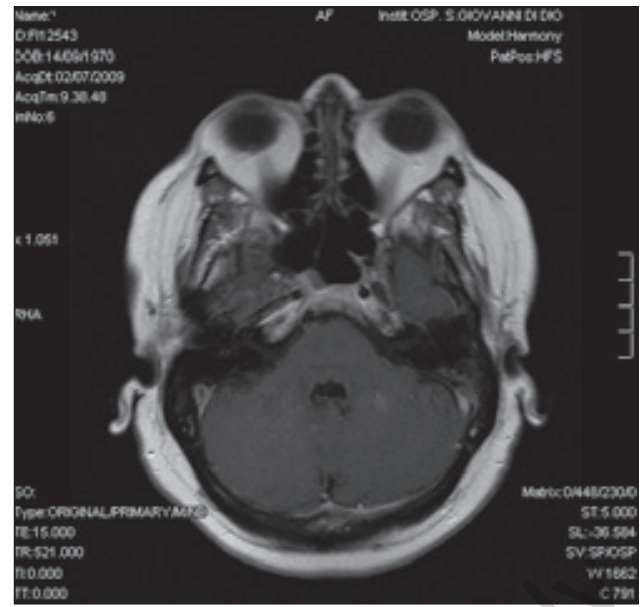

Figure 1 - MRI: dural tichening in the tentorium of the cerebellum.

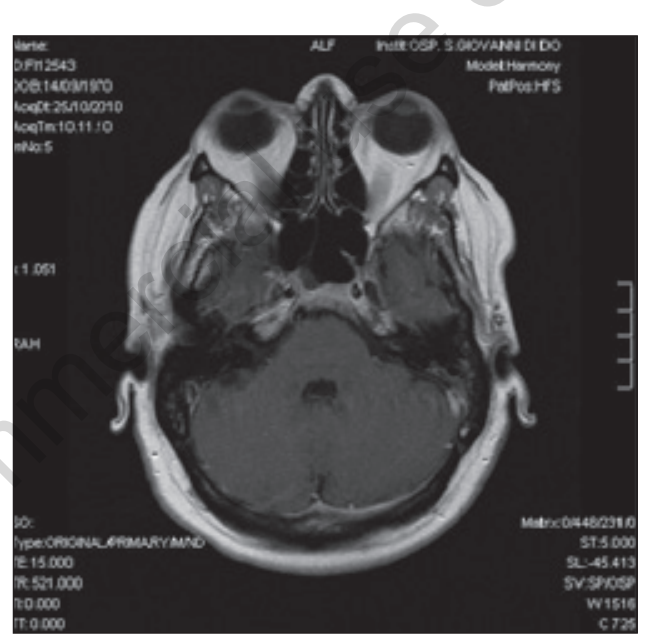

Figure 2 - MRI: decrease in the dural tichening in the tentorium of the cerebellum.

In January 2010, the patient was treated with prednisone $50 \mathrm{mg} /$ day combined with MTX $15 \mathrm{mg} /$ week and RTX $700 \mathrm{mg}$ (375 $\mathrm{mg} / \mathrm{m}^{2}$ ) per month for six months, and then every three months.

Two months later, the neurological symptoms improved with completed resolution at fourth month. MRI in October 2010 revealed a decrease in the dural thickening in the tentorium of the cerebellum (Fig. 2). Based on re-population of B lymphocytes, in particular by mean of flow-cytometry we evaluated the levels of naïve and memory CD27, the panels of B (CD20, CD38, $\mathrm{CD} 19)$, and we re-treated the patient every six-months. 


\section{DISCUSSION}

Chronic meningitis is a rare complication of GPA. It was detected in only two out of 324 GPA patients attending the Mayo Clinic in Rochester $(12,13)$ and in none of the patients described in other large series (14). Furthermore, a search for individual case reports revealed only 46 patients (15). Headache is the most frequent symptom associated with chronic meningitis but, as it is also associated with GPA (due to chronic sinusitis or orbital disease), meningeal involvement may remain unrecognised for a long time although, later in the course of the disease, most of patients develop other neurological abnormalities. The most frequently observed MRI finding is diffuse (followed by focal) dural thickening, usually involving the tentorium; the leptomenges are affected in only a minority of cases. Although $62.9 \%$ of the patients are positive for anti-neutrophil cytoplasmic antibodies (ANCAs), a cytoplasmic staining is observed in only $37.1 \%$ (16).

The majority of patients with GPA and meningeal involvement have been treated with high-dose corticosteroids and immunosuppressants. CYC is the most frequently used drug and, like MTX, leads to successful results. Every consensus suggests that organ involvement in GPA should be treated promptly and aggressively and, on the basis of the published data, high-dose of corticosteroids associated with CYC or MTX should be used for treating meningitis in GPA patients (15).

RTX is a chimeric monoclonal antibody against CD20, a B cell marker that is expressed from the pre- $\mathrm{B}$ to the mature phase of $\mathrm{B}$ cell development, but is not expressed in pro-B cells, memory cells, plasma cells, or antibody-secreting B cells. RTX binds CD20 in B cells and induces cell death by means of a still unknown mechanism, although in vitro experiments have shown that antibody-dependent cytotoxicity, complement-mediated B-cell lysis, apoptosis, and sensitisation to cytotoxic agents or corticosteroids may all play a role.

RTX has been used as rescue medication in patients with refractory GPA or contra- indications to CYC. About 100 published cases of GPA have been treated with RTX (17), which seems to be effective in treating its vasculitic manifestations such as pulmonary capillaritis and necrotising glomerulonephritis. However, it is still unclear whether RTX is equally effective in treating the necrotising granulomatous manifestations of GPA, such as orbital pseudotumours or subglottic stenosis (18). The Rituximab for ANCA-associated Vasculitis (RAVE study) (19), which involved 197 ANCA-positive patients with GPA or microscopic polyangiitis, found that RTX therapy was not inferior to daily CYC treatment in inducing remission and that it may be superior in relapsing disease. The results showed that $34 / 51$ patients in the RTX group $(67 \%)$ reached the primary end point against $21 / 50$ patients in the control group (42\%); moreover, only one patient in the RTX group had meningeal involvement. The Rituximab versus Cyclophosphamide in ANCA-Associated Renal Vasculitis (RITUXVAS) is a randomised controlled trial that included 44 patients with new diagnosed ANCA-associated vasculitis and renal involvement, who were randomised 3:1 to receive a standard glucocorticoid regimen plus four intravenous administrations of RTX $375 \mathrm{mg} /$ $\mathrm{m}^{2}$ per week, with two intravenous CYC pulses given concomitantly with the first and third RTX infusion, or the same standard glucocorticoid regimen plus intravenous CYC pulses for 3-6 months followed by oral azathioprine. The 12-month rate of sustained remissions (defined as a Birmingham vasculitis activity score of 0 for six months) was similar in both treatment arms (20).

Despite these important trials, a recent study involving 59 patients found that the overall response rate to RTX was high in patients with granulomatosis with polyangiitis $(61.3 \%$ experienced remission or improvement), but the patients with pachymeningitis or orbital involvement had the same rate of refractory disease $(33.3 \%)$ (21). The possible differences in term of response to RTX therapy among orbital involvement linked to granulomatous lesion 
and pachimengitis linked at vasculitis lesion are described (18).

A total of 7 cases that showed the efficacy of RTX in meningitis within GPA are described (22-24).

Patients with active vasculitis have a lower proportion of $\mathrm{Bm} 1$ cells that may indicate activated $B$ cells, whereas patients in remission have higher proportions of $\mathrm{CD} 25+$ (the $\alpha$-chain of the interleukin 2 receptor) and CD86+ (co-stimulatory molecule) B cells. This suggests that $B$ cells may play a regulatory role in the pathogenesis of GPA (25). ANCAs induce the release of BLyS from activated neutrophils that support B cell survival in vitro, and the presence of BLyS in the serum of patients with active disease and after B cell depletion suggests that it plays a role in B cell activity and survival (26). All of these findings support the use of RTX in patients with WG, and may explain possible post-treatment relapses. To the best of our knowledge, ours is the first case showing an improvement in neurological symptoms confirmed by means of cerebral MRI in a patient with WG and meningeal involvement after treatment with RTX combined with MTX and prednisone.

In conclusion, RTX therapy is an optimal means of treating CYC-resistant patients with WG associated with meningeal involvement. Our results justify its use in a large clinical series of such patients in order to confirm its efficacy.

\section{REFERENCES}

1. Jennette JC, Falk RJ, Andrassy K, et al. Nomenclature of systemic vasculitides. Proposal of an international consensus conference. Arthritis Rheum. 1994; 37: 187-92.

2. Fauci AS, Katz P, Haynes BF, Wolff SM. Cyclophosphamide therapy of severe systemic necrotizing vasculitis. N Engl J Med. 1979; 301: 235-8.

3. Hoffman GS, Kerr GS, Leavitt RY, et al. Wegener granulomatosis: an analysis of 158 patients. Ann Intern Med. 1992; 116: 488-98.

4. Sneller MC, Hoffman GS, Talar-Williams C, et al. An analysis of forty-two Wegener's granulomatosis patients treated with methotrexate and prednisone. Arthritis Rheum. 1995; 38: 608-13.
5. Guillevin L, Cordier JF, Lhote F, et al. A prospective, multicenter, randomized trial comparing steroids and pulse cyclophosphamide versus steroids and oral cyclophosphamide in the treatment of generalized Wegener's granulomatosis. Arthritis Rheum. 1997; 40: 2187 98.

6. Reinhold-Keller E, Fink COE, Herlyn K, et al. High rate of renal relapse in 71 patients with Wegener's granulomatosis under maintenance of remission with low-dose methotrexate. Arthritis Rheum. 2002; 47: 326-32.

7. de Groot K, Rasmussen N, Bacon PA, et al. Randomized trial of cyclophosphamide versus methotrexate for induction of remission in early systemic antineutrophil cytoplasmic antibody-associated vasculitis. Arthritis Rheum. 2005; 52: 2461-9.

8. Langford CA, Talar-Williams C, Barron KS, Sneller MC. A staged approach to the treatment of Wegener's granulomatosis: induction of remission with glucocorticoids and daily yclophosphamide switching to methotrexate for remission maintenance. Arthritis Rheum. 1999; 42: 2666-73.

9. Jayne D, Rasmussen N, Andrassy K, et al. A randomised trial of maintenance therapy for vasculitis associated with antineutrophil cytoplasmic autoantibodies. New Engl J Med. 2003; 349: 36-44.

10. Senolt L, Vencovsky J, Pavelka K, et al. Prospective new biological therapies for rheumatoid arthritis. Autoimmun Rev. 2009; 2: 102-7.

11. Leavitt RY, Fauci AS, Bloch DA, et al. The American College of Rheumatology 1990 criteria for the classification of Wegener's granulomatosis. Arthritis Rheum. 1990; 33: 1101-7.

12. Nishino H, Rubino FA, Deremee RA, et al. Neurological involvement in Wegener's Granulomatosis: an analysis of 324 consecutive patients at the Mayo Clinic. Ann Neurol. 1993; 33: 4-9.

13. Nishino H, Rubino FA, Parisi JE. The spectrum of neurological involvement in Wegener's granulomatosis. Neurology. 1993; 43: 1334-7.

14. Stone JH. Wegener's Granulomatosis Etanercept Trial Research Group. Limited versus severe Wegener's granulomatosis: baseline data on patients in the Wegener's granulomatosis etanercept trial. Arthritis Rheum. 2003; 48: 2299-309.

15. Di Comete G, Bozzolo EP, Praderio L, et al. Meningeal involvement in Wegener's granulomatosis is associated with localized disease. Clin Exp Rheum. 2006; 24: S60-4.

16. Murphy JM, Gomez-Anson B, Gillard JH. Wegener's granulomatosis: MR imaging findings in brain and meninges. Radiology. 1999; 213: 794-9.

17. Cartin Ceba R, Fervenza FC, Specks U. Treatment of antineutrophil cytoplasmic antibody 
associated vasculitis with Rituximab. Curr Op Rheum. 2012; 24: 15-23.

18. Aries PM, Hellmich B, Voswinkel J. Lack of efficacy of rituximab in Wegener's granulomatosis with refractory granulomatous manifestations. Ann Rheum Dis. 2006; 65: 853-8.

19. Stone JH, Merkel PA, Spiera R, et al. for RAVE-ITN Research Group. Rituximab versus cyclophosphamide for ANCA-associated vasculitis N Eng J Med. 2010; 363: 221-32.

20. Jones RB, Tervaert JW, Hauser T, et al. Rituximab versus cyclophosphamide in ANCA-associated renal vasculitis. N Engl J Med. 2010; 363: 211-20.

21. Holle JU, Dubrau C, Herlyn K, et al. Rituximab for refractory granulomatosis with polyangiitis (Wegener's granulomatosis): comparison of efficacy in granulomatous versus vasculitic manifestations. Ann Rheum Dis. 2012; 71: 327-33.

22. Bawa S, Mukhtyar C, Edmonds S, Webley M.
Refractory Wegener's meningitis treated with rituximab. J Rheumatol. 2007; 34: 900-1.

23. Just SA, Knudsen JB, Nielsen MK, Junker $\mathrm{P}$. Wegener's granulomatosis presenting with pachymeningitis: clinical and imaging remission by rituximab. ISRN Rheumatol. 2011; 201: 608-942.

24. Sharma A, Kumar S, Wanchu A, et al. Successful treatment of hypertrophic pachymengitis in refractory Wegener's granulomatosis with rituximab. Clin Rheumatol. 2010; 29: 107-10.

25. Eriksson P, Sandel C, Backteman K, Ernerudh J. B cells abnormalities in Wegener's granulomatosis and microscopic polyangiitis role of CD25+ expressing B cells. J Rheumatol. 2010; 37: 2086-95.

26. Holden NJ, Williams JM, Morgan MD, et al. ANCA-stimulated neutrophils release BLyS and promote B cell survival: a clinically relevant cellular process. Ann Rheum Dis. 2011; 70: 2229-33. 